\title{
Simulating operational control of production in lumber house building businesses
}

\author{
Dmitriy Korolchenko ${ }^{1}$, and Andrey Pizhurin ${ }^{1, *}$ \\ ${ }^{1}$ Moscow state university of civil engineering, Yaroslavskoye shosse, 26, Moscow, Russia, 129337
}

\begin{abstract}
Substantial reserves for higher operation efficiency in wooden house building businesses exist in improvements of production control system through methods of simulation and optimization. This report presents analysis of key factors of lumber house building processes that influence approaches to production control simulation. The need to factor in interconnection and dependence of lumber and house part production processes is highly difficult for operational control of the production system in question. The optimization mathematical models that we developed will help to solve the tasks of operational scheduling at all stages of wooden house manufacturing, from log cutting to production of finished assembly sets.
\end{abstract}

\section{Introduction}

The current economic situation in the wooden housebuilding sector in Russia is experiencing a shortage of funds for radical upgrade of production equipment, as well as persistent deficit of working capital. The market forces businesses to expand their product ranges and move on to small-scale production and to customer-built houses. All this calls for improved control methods, primarily ensuring flexible and optimal production processes in real time [1].

Under the circumstances, businesses are confronted with difficult problems of ensuring timely deliveries to users, well-paced manufacturing, optimized equipment load distribution, creating regulated reserves, balanced production plans and resources, ensuring strict synchronization of warehouses, motor vehicles and equipment, production facilities and operation sites [2].

Solution of the above tasks should be addressed out by a production control system built with optimization mathematical models.

There have been numerous studies to develop efficient operation control systems, and such research goes well back in time [3-9]. However, studies of operation control in woodworking business are rather few, and those available fail to exhaustively cover the capability of today's information technologies and production control system simulation methods. Besides, most of such studies mainly focus on operation management of sawmills [10-14], pulp-and-paper mills [15, 16] or furniture manufacturing businesses [17].

* Corresponding author: ananpi@yandex.ru 
The main purpose of this paper is to design mathematical optimization models addressing issues of time operation planning for multi-stage processes of wooden house building.

\section{Problem description}

As its operation control object, this study examines production of wooden houses that uses processes to transform lumber and other materials and power to semi-finished and finished products within a complex business. As the key feature, each such business must include sawmills and woodworking units to manufacture joinery products, wooden structures, and wooden houses assembly sets. Such production system is a complex one because both a sawmill and a woodworking shop use highly complicated processes, and when they are integrated into a united interrelated production process the system's complexity rises to a whole new level. Wooden house manufacturing is a multi-stage process that includes lumber shaping to finished-product set delivery or on-site assembly. The stages of processing are handled by a central logistical system with many warehouses to accumulate stock between steps (saw logs, sawn lumber, blanks, parts, and assembly units).

Unlike in other industries, material is a major extra randomization factor for the production environment of wooden house building, because as a product of living nature, wood is highly diverse and unpredictable in terms of dimensional and qualitative characteristics.

One of the main objectives for production control systems is to achieve coordinated control of sawmilling and woodworking in the context of supply of lumber, blanks and parts within the premises, and thus organizing a comprehensive production process.

\section{Modelling}

To work out some rational managerial solutions, a wooden house building business needs to prepare mathematical models addressing the problems of optimized time scheduling for each stage of house manufacturing, from lumber cutting to specific finished parts.

The task of operation time scheduling for log sawing is stated as follows.

The sawmill uses $L$ parallel production flows to process logs. Let $l=1 \ldots \ldots L$ be the numbers of the process flows.

Timber logs are sorted to form $M$ dimension groups. Let $m=1, \ldots, M-$ be the numbers of the log size groups.

A variety of cutting patterns can be used to mill the timber. Total number of usable rational cutting patterns is $P$. Let then $p=1, \ldots, P$ be the serial number of any pattern in the list of cutting patterns.

Specification for lumber to be produced during the planned time period contains $K$ of various type-sizes of boards. Let $k=1, \ldots, K$ be the number of lumber type-sizes in the specification.

The entire scheduled operation time period is divided into $T$ time intervals of equal length, such as half-shift. Let $t=1, \ldots, T$ is the serial number of the respective planning interval (control step).

For the planning period, we set the deadlines $\tau_{k}$ and volumes $V_{k}$, of lumber of $k$-th typesize, to be produced by the deadlines. We also assume permissible above target $V_{k}^{+}$and below target $V_{k}^{-}$for production of $k$-th type-size lumber during the planning period.

We know number $Q_{m}$ of logs in each $m$-th group, stored at the stock warehouse during the planning period. 
During the same interval of the planning period, each flow may only use one mill (cutting shape) to process material.

Total quantity of type-size lumber produced during each interval of the planning period is limited by number $N$ of pallets in the sorting bay (or recesses in the sorting line). In addition, some pairs of lumber type-sizes exist that are illegal for simultaneous production, because they are hard to tell apart during sorting. We assume that we have a total $S$ of such pairs, while $s=1, \ldots, S$ is the serial number of the pair in the list. Let us take that $\Omega_{s}$ is a set of numbers of lumber type-sizes, which stands for a pair illegal for simultaneous production.

Now we need to generate a calendar time schedule for stock milling: we therefore need to know which group of logs will be milled during each interval of the planning period, using which cutting pattern and as part of which flow. The following constraints of technology and planning apply.

The quality of any time schedule is decided by two criteria. First, economic criterion $F_{1}$ requires maximized total cost of all production output milled during the planning period. Second, technological criterion $F_{2}$ represents the requirement of least changes in the product range during the planning period, helping to rapidly accumulate batches of boards in the same type-size and raise equipment load efficiency during lumber drying and finishing.

Let us adopt the following notation:

$x_{l m p}^{t}$-the problem's variable that is 1 , if during the $t$-th planning period in $l$-th flow to mill the material of $m$-th group using $p$-th cutting pattern; else the value is 0 .

$a_{k m p}-$ quantity of $k$-th type-size lumber, made of logs of $m$-th group using $p$-th cutting pattern.

$b_{l m p}$ - output capacity of $l$-th flow while milling $\operatorname{logs}$ of $m$-th group using $p$-th cutting pattern during the planning interval.

$C_{k}$ - cost of a unit of lumber of $k$-th type-size;

$\operatorname{sgn}(d)$ - sign $d$ - function defined as:

$\operatorname{sgn}(d)=\left\{\begin{array}{l}1, \text { if } d>0 \\ 0, \text { if } d \leq 0 .\end{array}\right.$

Now the mathematical model for the task of generating an optimal time schedule for material milling will appear as follows.

Criteria of optimization:

$$
\begin{gathered}
F_{1}=\max \sum_{t=1}^{T} \sum_{k=1}^{K} \sum_{l=1}^{L} \sum_{m=1}^{M} \sum_{p=1}^{P} C_{k} a_{k m p} b_{l m p} x_{l m p}^{t} \\
F_{2}=\min \left\{\sum_{t=1}^{T-1} \sum_{k=1}^{K}\left|\operatorname{sgn}\left(\sum_{l=1}^{L} \sum_{m=1}^{M} \sum_{p=1}^{P} a_{k m p} b_{l m p} x_{l m p}^{t}\right)-\operatorname{sgn}\left(\sum_{l=1}^{L} \sum_{m=1}^{M} \sum_{p=1}^{P} a_{k m p} b_{l m p} x_{l m p}^{t}\right)\right|\right\} .
\end{gathered}
$$

Limitations:

1. volume of material to mill

$$
\sum_{t=1}^{T} \sum_{l=1}^{l} \sum_{p=1}^{P} b_{l m p} x_{l m p}^{t} \leq Q_{m}, m=1, \ldots, M ;
$$

2. volume of lumber to produce

$$
V_{k}-V_{k}^{-} \leq \sum_{t=1}^{\tau_{k}} \sum_{l=1}^{L} \sum_{m=1}^{M} \sum_{p=1}^{P} a_{k m p} b_{l m p} x_{l m p}^{t} \leq V_{k}+V_{k}^{+}, k=1, \ldots, K
$$

3. number of lumber type-sizes produced simultaneously 


$$
\sum_{k=1}^{K} \operatorname{sgn}\left(\sum_{l=1}^{L} \sum_{m=1}^{M} \sum_{p=1}^{P} a_{k m p} b_{l m p} x_{l m p}^{t}\right) \leq N, \quad t=1, \ldots, T ;
$$

4. individual lumber type-sizes produced simultaneously

$$
\sum_{k \in \Omega_{s}} \operatorname{sgn}\left(\sum_{l=1}^{L} \sum_{m=1}^{M} \sum_{p=1}^{P} a_{k m p} b_{l m p} x_{l m p}^{t}\right) \leq 1, s=1, \ldots, S ; t=1, \ldots, T ;
$$

5. use of only one milling pattern for each flow during any single planning interval

$$
\sum_{m=1}^{M} \sum_{p=1}^{P} x_{l m p}^{t}=1, l=1, \ldots, L ; t=1, \ldots, T
$$

6. variables of the mathematical model

$$
x_{l m p}^{t}=1 \vee 0, p=1, \ldots P ; m=1, \ldots, M ; l=1, \ldots, L ; t=1, \ldots, T .
$$

The production process to make assembly sets of wooden houses from lumber is treated as a multi-stage line production system. The system includes the stages of lumber patterns cutting to make blanks, blank processing to make parts, and preparing sets of parts as per respective specification. Certain cases, depending on the wooden house design, may include the stage that assembles wooden panels. Each such stage can include multiple steps of blank and part processing. The number of steps and the sequence for the blanks and parts to follow through depend on engineering and art design of the housing product, and the technology adopted by the business. Manipulations of the production process can be performed on several machines working in parallel. To coordinate operation of various stages and steps of the production process, the business must establish in-process stocks to store blanks. Such stock enables control of all blanks passing each processing stage, because blanks can enter a specific stage from both the previous processing stage and from the warehouse. The blank/part processing stage is simulated as an in-process stock control system. For ongoing control of the production process, the business must draw a time schedule of operations to plan for changes in sizes of in-process stock with time.

Let us examine the problem of operation time schedule planning for the woodworking stages.

We adopt the following notation:

$T$ - planning horizon - planned time interval expressed in work shifts;

$t$ - number of planned work shift, $t=1, \ldots, T$;

$m$ - number of work shift in the planned period, $m=1, \ldots, T$;

$L-$ total number of blank/part processing stages at the business;

$l-$ serial number of processing stage, $l=1, \ldots, \mathrm{L}$;

$R^{(l)}$ - number of machines/lines in $l$-th processing stage, used in parallel;

$r$-serial number of machine/line in $l$-th processing stage, $r=1, \ldots, R^{(l)}$;

$T_{r}^{(l)}$-effective run time of each $r$-th machine/line in $l$-th stage;

$N$ - total number of wooden house projects / products made by the business;

$j$ - number of project/product in the business's product range, $j=1, \ldots, N$;

$M$ - total number of type-sizes of parts/blanks made by the business;

$i$ - number of part/blank as per the manufacturer's general specification, $i=1, \ldots, M$;

$a_{i j}$ - number of parts/blanks of $i$-th type-size in $j$-th product;

$W_{1 i}^{(l)}$ - number of $i$-th type-size blanks existing before $l$-th processing stage at the beginning of the planning period;

$W_{2 i}^{(l)}$ - number of $i$-th type-size blanks existing after $l$-th processing stage at the beginning of the planning period; 
$Q_{1 i}^{(l)-}, Q_{1 i}^{(l)+}-$ least/greatest number of $i$-th type-size part/blank that may be stored before processing at $l$-th stage;

$Q_{2 i}^{(l)-}, Q_{2 i}^{(l)+}-$ least/greatest number of $i$-th type-size part/blank that may be stored after processing at $l$-th stage;

$S_{1}^{(l)}, S_{2}^{(l)}$-warehouse space before and after $l$-th processing stage;

$H_{1}^{(l)}, H_{2}^{(l)}$ - warehouse storage capacity coefficients before and after $l$-th processing stage;

$s_{i}$ - area of i-th type-size part/blank;

$h_{i}-$ number of i-th type-size part/blank in the stack;

$\tau_{i r}^{(l)}$ - time to process i-th type-size part/blank by $r$-th machine/line of $l$-th stage;

$\tau_{r}^{(l)}$ - time to readjust $r$-th machine/line of $l$-th stage;

$N_{\max r}^{(l)}$-possible greatest number of readjustments of $r$-th machine/line of $l$-th stage per work shift;

$3_{i r}^{(l)}-$ cost to process one $i$-th type-size blank by $r$-th machine/line of $l$-th stage;

$3_{1 i}^{(l)}, 3_{2 i}^{(l)}$ - storage cost per $i$-th type-size blank warehouses respectively before and after $l$-th stage during the work shift.

Let us introduce the following variables into our mathematical model:

$\lambda_{\text {irm }}^{(l)}=\left\{\begin{array}{l}1, \text { if } i \text {-th type-size blanks are processed by } r \text {-th machine of } l \text {-th stage } \\ \quad \text { during } m \text {-th shift } \\ 0, \text { otherwise; }\end{array}\right.$

$x_{i r m}^{(l)}$-number of $i$-th type-size blanks processed during $m$-th shift by $r$-th machine of $l$-th stage;

$V_{i m}^{(l)}-$ number of $i$-th type-size blanks, entering $l$-th stage from previous stages during $m$-th shift;

$U_{i m}^{(l)}-$ number of $i$-th type-size blanks, exported from $l$-th stage to subsequent processing stages during $m$-th shift.

The resulting mathematical model for the problem of operation time schedule planning to manufacture assembly part sets of wooden houses for $l$-th processing stage appears as follows:

The target function of the problem is to minimize total costs of blank processing and storage at warehouses in between operations:

$$
\begin{aligned}
F_{3}= & \left\{\sum_{m=1}^{T} \sum_{r=1}^{R^{(l)}} \sum_{i=1}^{M} 3_{i r}^{(l)} \lambda_{i r m}^{(l)} x_{i r m}^{(l)}+\sum_{t=1}^{T} \sum_{i=1}^{M} 3_{1 i}^{(l)} \cdot\left(W_{1 i}^{(l)}+\sum_{m=1}^{t}\left(V_{i m}^{(l)}-\sum_{r=1}^{R^{(l)}} \lambda_{i r m}^{(l)} x_{i r m}^{(l)}\right)\right)+\right. \\
& \left.+\sum_{t=1}^{T} \sum_{i=1}^{M} 3_{2 i}^{(l)} \cdot\left(W_{2 i}^{(l)}+\sum_{m=1}^{t}\left(\sum_{r=1}^{R^{(l)}} \lambda_{i r m}^{(l)} x_{i r m}^{(l)}-U_{i m}^{(l)}\right)\right)\right\} \rightarrow \text { min. }
\end{aligned}
$$

Limitations:

1. number of blanks warehoused before processing of $l$-th stage

$$
Q_{1 i}^{(l)-} \leq W_{1 i}^{(l)}+\sum_{m=1}^{t}\left(V_{i m}^{(l)}-\sum_{r=1}^{R^{(l)}} \lambda_{i r m}^{(l)} x_{i r m}^{(l)}\right) \leq Q_{1 i}^{(l)+}, i=1, \ldots, M ; t=1, \ldots, T ;
$$


2. number of blanks warehoused after processing of $l$-th stage

$$
Q_{2 i}^{(l)-} \leq W_{2 i}^{(l)}+\sum_{m=1}^{t}\left(\sum_{r=1}^{R^{(l)}} \lambda_{i r m}^{(l)} x_{i r m}^{(l)}-U_{i m}^{(l)}\right) \leq Q_{2 i}^{(l)+}, i=1, \ldots, M ; t=1, \ldots, T ;
$$

3. capacity of stock warehouse before processing of $l$-th stage

$$
\sum_{i=1}^{M} \frac{s_{i}}{h_{i}}\left(W_{1 i}^{(l)}+\sum_{m=1}^{t}\left(V_{i m}^{(l)}-\sum_{r=1}^{R^{(l)}} \lambda_{i r m}^{(l)} x_{i r m}^{(l)}\right)\right) \leq H_{1}^{(l)} S_{1}^{(l)}, t=1, \ldots, T ;
$$

4. capacity of stock warehouse after processing of $l$-th stage

$$
\sum_{i=1}^{M} \frac{s_{i}}{h_{i}}\left(W_{2 i}^{(l)}+\sum_{m=1}^{t}\left(\sum_{r=1}^{R^{(l)}} \lambda_{i r m}^{(l)} x_{i r m}^{(l)}-U_{i m}^{(l)}\right)\right) \leq H_{2}^{(l)} S_{2}^{(l)}, t=1, \ldots, T ;
$$

5. output of machines used to process blanks during $l$-th stage per shift

$$
\sum_{i=1}^{M}\left(\tau_{i r}^{(l)} x_{i r m}^{(l)}+\tau_{r}^{(l)}\right) \lambda_{i r m}^{(l)} \leq T_{r}^{(l)}, r=1, \ldots, R^{(l)} ; m=1, \ldots, T ;
$$

6. readjustment cycles for machines used to process blanks during $l$-th stage per shift

$$
\sum_{i=1}^{M} \lambda_{i r m}^{(l)} \leq N_{\max r}^{(l)}, \quad r=1, \ldots, R^{(l)} ; m=1, \ldots, T ;
$$

7. correlation of variables

$$
\begin{gathered}
x_{i r m}^{(l)} \in Z_{+} ; V_{i m}^{(l)} \in Z_{+} ; U_{i m}^{(l)} \in Z_{+} ; \lambda_{i r m} \in\{0 ; 1\}:\left(1-\lambda_{i r m}\right) x_{i r m}=0 \wedge\left(x_{i r m}-1\right) \lambda_{i r m} \geq 0 \\
i=1, \ldots, M ; r=1, \ldots, R^{(l)} ; m=1, \ldots, T .
\end{gathered}
$$

\section{Conclusions}

Mathematical analysis of the resulting models demonstrates that they can be reduced to the problem of unconditional pseudo-Boolean optimization with target functions preset algorithmically as a problem of conditional optimization with continuous or discrete variables. Coefficients for Boolean variables are not constant values, but they represent functions of the same Boolean variables. To solve this problem, we use a hybrid adaptive heuristic search algorithm.

The proposed set of interrelated mathematical models is basis for the designed system to support operational control of wooden house manufacturing.

Future studies are expected to consider the uncertainty of the production environment of the businesses of wooden house building using the methods of fuzzy mathematical programming.

\section{References}

1. A. S. Ermakov, O. G. Mukhamedzhanova, To the enlightened development of the construction industry, Integration, partnership and innovation in building science and education.The collection of materials of the International Scientific Conference, 329-333, (2017)

2. B. Skołud, Market oriented approach to the production management on the operational level, Adv. Mater. Res., 837, 663-668, (2014)

3. K. N. McKay, G. W. Black, The evolution of a production planning system: A 10-year case study, Comput. Ind., 58(8-9), 756-771, (2007) 
4. C. T. Maravelias, C. Sung, Integration of production planning and scheduling: Overview, challenges and opportunities, Comput. Chem. Eng., 33(12), 1919-1930, (2009)

5. I. C. Dima, M. Man, Managerial Systems, Methods, and Techniques Used in Scheduling Industrial Production, Industrial Production Management in Flexible Manufacturing Systems (IGI Global, 345-369, 2013)

6. I. Harjunkoski, C. T. Maravelias, P. Bongers, P. M. Castro, S. Engell, I. E. Grossmann, J. Hooker, C. Méndez, G. Sand, J. Wassick, Scope for industrial applications of production scheduling models and solution methods, Comput. Chem. Eng., 62, 161-193, (2014)

7. M. Baldea, I. Harjunkoski, Integrated production scheduling and process control: A systematic review, Comput. Chem. Eng., 71, 377-390, (2014)

8. D. A. Korolchenko, E. N. Degaev, A. F. Sharovarnikov, Dependence of Fire Extinguishing Efficacy of Low Expansion Foams Solutions Homology Sodium Sulfate on the Molecular Weight of the Surface-active Substances, 2nd International conference on material engineering and application (ICMEA 2015)

9. D. Korolchenko, S. Voevoda, Influence of spreading structure in an aqueous solutionhydrocarbon system on extinguishing of the flame of oil products, MATEC Web of Conferences, 86, (2016)

10. S. Maturana, E. Pizani, J. Vera, Scheduling production for a sawmill: A comparison of a mathematical model versus a heuristic, Comput. Ind. Eng., 59(4), 667-674, (2010)

11. M. K. Zanjani, M. Nourelfath, D. Ait-Kadi, Sawmill Production Planning Under Uncertainty: Modelling and Solution Approaches, Stochastic Programming, 347-395, (2012)

12. M. K. Zanjani, D. A. Kadi, M. Nourelfath, A stochastic programming approach for sawmill production planning, IJMOR, 5(1), 1, (2013)

13. M. Varas, S. Maturana, R. Pascual, I. Vargas, J. Vera, Scheduling production for a sawmill: A robust optimization approach, Int. J. Prod. Econ., 150, 37-51, (2014)

14. A. Lobos, J. R. Vera, Intertemporal stochastic sawmill planning: Modeling and managerial insights, Comput. Ind. Eng., 95, 53-63, (2016)

15. D. Bredstrom, M. Ronnqvist, Integrated production planning and route scheduling in pulp mill industry, Proceedings of the 35th Annual Hawaii International Conference on System Sciences, (2002)

16. G. Figueira, P. Amorim, L. Guimarães, M. Amorim-Lopes, F. Neves-Moreira, B. Almada-Lobo, A decision support system for the operational production planning and scheduling of an integrated pulp and paper mill, Comput. Chem. Eng., 77, 85-104, (2015)

17. J. C. Vidal, M. Mucientes, A. Bugarín, M. Lama, Machine scheduling in custom furniture industry through neuro-evolutionary hybridization, Appl. Soft Comput., 11(2), 1600-1613, (2011) 